\title{
Phonon-Induced Exciton Dephasing in Quantum Dot Molecules
}

\author{
E. A. Muljarov, ${ }^{1,2,3, *}$ T. Takagahara, ${ }^{2}$ and R. Zimmermann ${ }^{1}$ \\ ${ }^{1}$ Institut für Physik der Humboldt-Universität zu Berlin, Newtonstrasse 15, D-12489 Berlin, Germany \\ ${ }^{2}$ Kyoto Institute of Technology, Matsugasaki, Sakyo-ku, Kyoto 606-8585, Japan \\ ${ }^{3}$ General Physics Institute, Russian Academy of Sciences, Vavilova 38, Moscow 119991, Russia
}

(Received 5 May 2005; published 21 October 2005)

\begin{abstract}
A new microscopic approach to the optical transitions in quantum dots and quantum dot molecules, which accounts for both diagonal and nondiagonal exciton-phonon interaction, is developed. The cumulant expansion of the linear polarization is generalized to a multilevel system and is applied to calculation of the full time dependence of the polarization and the absorption spectrum. In particular, the broadening of zero-phonon lines is evaluated directly and discussed in terms of real and virtual phononassisted transitions. The influence of Coulomb interaction, tunneling, and structural asymmetry on the exciton dephasing in quantum dot molecules is analyzed.
\end{abstract}

DOI: 10.1103/PhysRevLett.95.177405

PACS numbers: 78.67.Hc, 71.35. $-\mathrm{y}, 71.38 .-\mathrm{k}$

Semiconductor quantum dots (QDs) have been considered for several years as promising candidates to form an elementary building block (qubit) for quantum computing. Recently quantum dot molecules (QDMs), i.e., systems of two quantum-mechanically coupled QDs, have been proposed for realization of optically driven quantum gates involving two-qubit operations [1-3]. It turns out, however, that contrary to their atomic counterparts, QDs have a strong temperature-dependent dephasing of the optical polarization. Such a decoherence caused by the interaction of the electrons with the lattice vibrations (phonons) is inevitable in solid state structures thus presenting a fundamental obstacle for their application in quantum computing.

There has been considerable progress in the understanding of exciton dephasing in QDs after a seminal paper by Borri et al. on four-wave mixing measurements in InGaAs QDs [4]. Two important and well understood features of the dephasing are (i) the optical polarization experiences a quick initial decay within the first few picoseconds after pulsed excitation and (ii) at later times it shows a much slower exponential decay. In photoluminescence and absorption spectra this manifests itself as (i) a broadband and (ii) a much narrower Lorentzian zero-phonon line (ZPL) with a temperature-dependent linewidth [5]. Such a behavior of the polarization is partly described within the widely used independent boson model $[6,7]$ that allows an analytic solution for the case of a single exciton state. It describes satisfactorily the broadband (or the initial decay of the polarization). However, in this model there is no longtime decay of the polarization (no broadening of the ZPL).

Recently we have presented a first microscopic calculation of the ZPL width in single QDs [8], taking into account virtual phonon-assisted transitions into higher exciton states and mapping the off-diagonal linear excitonphonon coupling to a diagonal but quadratic Hamiltonian. This is the major mechanism of phonon-induced dephasing in single QDs as long as exciton level distances are much larger than the typical energy range of the acoustic pho- nons coupled to the QDs ( $\leq 3 \mathrm{meV}$ in InGaAs QDs). On the contrary, in QDMs the distance between certain exciton levels can be made arbitrarily small if the tunneling between dots is weak enough [9], so that the interaction with acoustic phonons can lead to real transitions (changing the level occupation). The experimentally measured excitonic polarization shows a different behavior in QDMs [10], too: the long-time decay is multiexponential, in contrast to a single-exponential one in uncoupled QDs [4].

To take into account both real and virtual transitions we develop in this Letter a new approach for a multilevel excitonic system which is coupled to acoustic phonons both diagonally and nondiagonally. This allows us to calculate the dephasing in QDMs, as well as the full timedependent linear polarization and absorption. Instead of the self-energy approach [11] which is more standard in the electron-phonon problem, we use the cumulant expansion $[6,12]$. It is much more advantageous when studying the dephasing and, being applied to a multilevel system, has to be generalized to a matrix form.

To calculate the linear polarization we reduce the full excitonic basis to the Hilbert space of single exciton states $|n\rangle$ [with bare transition energies $E_{n}$ and wave functions $\left.\Psi_{n}\left(\mathbf{r}_{e}, \mathbf{r}_{h}\right)\right]$. Then the exciton-phonon Hamiltonian takes the form

$$
\begin{aligned}
H= & \sum_{n} E_{n}|n\rangle\left\langle n\left|+\sum_{\mathbf{q}} \omega_{q} a_{\mathbf{q}}^{\dagger} a_{\mathbf{q}}+\sum_{n m} V_{n m}\right| n\right\rangle\langle m|, \\
V_{n m}= & \sum_{\mathbf{q}} M_{\mathbf{q}}^{n m}\left(a_{\mathbf{q}}+a_{-\mathbf{q}}^{\dagger}\right), \\
M_{\mathbf{q}}^{n m}= & \sqrt{\frac{\omega_{q}}{2 \rho_{M} u_{s}^{2} \mathcal{V}} \int d \mathbf{r}_{e} d \mathbf{r}_{h} \Psi_{n}^{*}\left(\mathbf{r}_{e}, \mathbf{r}_{h}\right) \Psi_{m}\left(\mathbf{r}_{e}, \mathbf{r}_{h}\right)} \\
& \times\left[D_{c} e^{i \mathbf{q} \mathbf{r}_{e}}-D_{v} e^{i \mathbf{q} \mathbf{r}_{h}}\right],
\end{aligned}
$$

where $a_{\mathbf{q}}^{\dagger}$ is the acoustic phonon creation operator, $D_{c(v)}$ is the deformation potential constant of the conduction (va- 
lence) band, $\rho_{M}$ is the mass density, $u_{s}$ the sound velocity, $\mathcal{V}$ the phonon normalization volume, and $\hbar=1$.

The linear polarization is given by

$$
P(t)=\langle\hat{\mu}(t) \hat{\mu}(0)\rangle=\sum_{n m} \mu_{n}^{*} \mu_{m} e^{-i E_{n} t} P_{n m}(t)
$$

where $\mu_{n}=\mu_{c v} \int d \mathbf{r} \Psi_{n}(\mathbf{r}, \mathbf{r})$ are the exciton matrix elements of the interband dipole moment operator $\hat{\mu}$. The components of the polarization are written as standard perturbation series,

$$
\begin{aligned}
P_{n m}(t)= & \sum_{k=0}^{\infty}(-1)^{k} \int_{0}^{t} d t_{1} \int_{0}^{t_{1}} d t_{2} \ldots \int_{0}^{t_{2 k-1}} d t_{2 k} \sum_{p r \ldots s} e^{i \Omega_{n p} t_{1}} \\
& \times e^{i \Omega_{p r} t_{2}} \ldots e^{i \Omega_{s m} t_{2 k}\left\langle V_{n p}\left(t_{1}\right) V_{p r}\left(t_{2}\right) \ldots V_{s m}\left(t_{2 k}\right)\right\rangle,}
\end{aligned}
$$

where the finite-temperature expectation value is taken over the phonon system, and the difference energies are $\Omega_{n m}=E_{n}-E_{m}$. The expansion of $\exp \left(-i E_{n} t\right) P_{n m}(t)$, which is in fact the full exciton Green's function, is shown diagrammatically in Fig. 1 up to second order, where the phonon Green's function $\left\langle\mathcal{T}^{\prime} V_{n k}(t) V_{p m}\left(t^{\prime}\right)\right\rangle$ (dashed lines) depends on four exciton indices.

Instead of the plain or self-energy summation of diagrams we introduce for each time $t$ the cumulant matrix $\hat{K}(t)$ defined as

$$
\hat{P}(t)=e^{\hat{K}(t)},
$$

where $\hat{P}(t)=\hat{1}+\hat{P}^{(1)}+\hat{P}^{(2)}+\cdots$ is the expansion of the polarization matrix $P_{n m}$ given by Eq. (4). Then the corresponding expansion for the cumulant is easily generated:

$$
\hat{K}(t)=\hat{P}^{(1)}+\hat{P}^{(2)}-\frac{1}{2} \hat{P}^{(1)} \hat{P}^{(1)}+\cdots .
$$

Numerically, we restrict ourselves to a finite number of exciton levels and diagonalize the cumulant matrix $\hat{K}$ at a given time in order to find the polarization via Eq. (5).

If all off-diagonal elements of the exciton-phonon interaction are neglected $\left(M_{\mathbf{q}}^{n m}=\delta_{n m} M_{\mathbf{q}}^{n n}\right)$, the cumulant expansion ends already in first order: the contribution of all higher terms of the polarization is exactly cancelled in the cumulant, Eq. (6), by lower order products. This result allows the exact solution of the independent boson model [6]. The inclusion of the nondiagonal interaction leads to nonvanishing terms in the cumulant in any order. Still, there is a partial cancellation of diagrams which provides a large time asymptotics of the cumulant, $\hat{K}(t) \rightarrow-\hat{S}-$ $i \hat{\omega} t-\hat{\Gamma} t$, that is linear in time. Consequently, the line

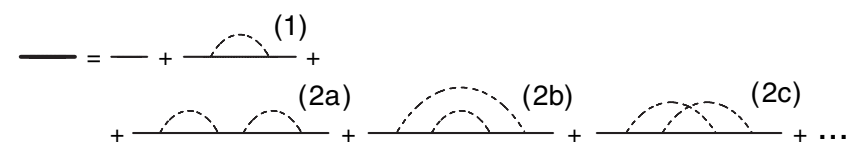

FIG. 1. Diagram representation of the perturbation series for the full exciton Green's function up to second order. shape of the ZPL is Lorentzian. For example, diagrams (1), (2b), and (2c) in Fig. 1 behave linear in time at $t \gg L / u_{s}$ ( $L$ is the QD size), while diagram (2a) in Fig. 1 has a leading $t^{2}$ behavior. In the cumulant, however, this quadratic term is cancelled exactly by the square of diagram (1) in Fig. 1, $\hat{P}^{(1)} \hat{P}^{(1)} / 2$.

The broadening of the ZPL (which is absent in the independent boson model) is exclusively due to the nondiagonal exciton-phonon interaction and appears already in first order of the cumulant. Remarkably, the cumulant expansion reproduces in first order exactly Fermi's golden rule for real phonon-assisted transitions: $\Gamma_{1}^{(1)}=$ $\pi N_{\text {Bose }}(\Delta E) \sum_{\mathbf{q}}\left|M_{\mathbf{q}}^{12}\right|^{2} \delta\left(\Delta E-\omega_{q}\right)$, where the ground state dephasing rate $\Gamma_{1}^{(1)}$ is given here for a system with two excitonic levels only. It is calculated for a single spherical QD as a function of the level distance $\Delta E=E_{2}-E_{1}$ (Fig. 2, dashed curve). As $M_{\mathbf{q}}^{12}$ decays with $\mathbf{q}$ due to the localization of the exciton wave functions (Gauss type in the present model calculation), $\Gamma_{1}^{(1)}$ also decays quickly with $\Delta E$ (see the parabola in the logarithmic scale). It exhibits a maximum at $\Delta E=\omega_{0} \sim u_{s} / L$ which is a typical energy of phonons coupled to the QD.

In spite of this "phonon bottleneck" effect, virtual transitions are always present in QDs due to second-order diagrams (2b) and (2c) in Fig. 1 and lead to a nonvanishing broadening of the ZPL everywhere. They have been taken into account already within our quadratic coupling model [8] which is valid in the opposite limit $\Delta E \gg \omega_{0}$ (dashdotted curve in Fig. 2). In the present calculation we do much better than in Ref. [8]: we account for both real and virtual transitions on an equal footing (up to second order in the cumulant) and cover the full range of possible values of exciton level distances (Fig. 2, full curve).

To describe excitonic states in a QDM, we restrict ourselves in the present calculation to a four-level model.

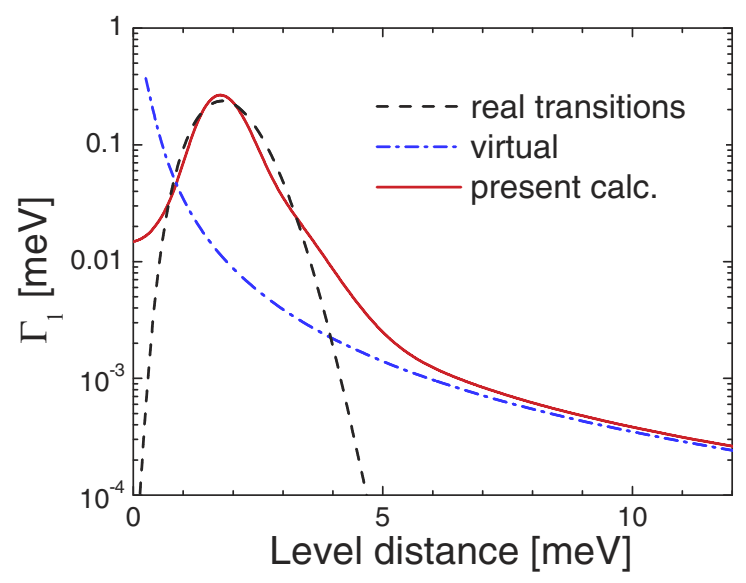

FIG. 2 (color online). Broadening of the ground state ZPL as a function of the exciton level distance $E_{2}-E_{1}$ in a spherical InGaAs QD, calculated accounting for real transitions (in first order), virtual transitions (according to Ref. [8]) and for both real and virtual transitions up to second order in the cumulant. 
We take into account for electron and hole the two lowest localized states each. Without Coulomb interaction the electron-hole pair state is a direct product of the oneparticle states which form our basis of four states. Then we include the Coulomb interaction and diagonalize a four-by-four Hamiltonian. Such a four-level model is valid as far as the Coulomb matrix elements are smaller than the energetic distances to higher confined ( $p$ shell) or wetting layer states.

Since a strictly symmetric QDM would lead by degeneracy to special features [9], we concentrate here on a slightly asymmetric situation: the confining potentials of the left dot are $2 \%$ deeper than those of the right one. This is close to the realistic situation, when the In concentration fluctuates from dot to dot. At the same time, the shape fluctuations of the QDM are less important for shallow QDs studied in the experiments $[1,3,10]$. Thus we assume both dots to have the same cylindrical form with height $L_{z}=1 \mathrm{~nm}$ (in the growth direction) adjusted from the comparison with experimentally measured transition energies $[1,10]$ (taking $92 \%$ of In concentration). Given that $s$, $p, d$, and $f$ shells in the luminescence spectra of QDMs have nearly equidistant positions [13], the in-plane confining potentials are taken parabolic with Gaussian localization lengths of carriers adjusted to $l_{e}=6.0 \mathrm{~nm}$ and $l_{h}=$ $6.5 \mathrm{~nm}$ [14]. The electronic band parameters are taken from Ref. [15] and the acoustic phonon parameters are the same as used previously [8].

While in single dots the Coulomb interaction results in a small correction to the polarization decay, in QDMs the exciton wave functions (and consequently $M_{\mathbf{q}}^{n m}$ ) are strongly affected by the Coulomb energy [9]. Even more important is the influence of the Coulomb interaction and asymmetry of the QDM on the exciton transitions energies shown in Fig. 3(a) in dependence on (center-to-center) dot distance $d$.

At short distances $d$ the tunneling exceeds both the Coulomb energy and the asymmetry, and the exciton states are well described in terms of one-particle states. Like in symmetric QDMs [9], optically active states $|1\rangle=|S S\rangle$ and $|4\rangle=|A A\rangle$ are formed from, respectively, symmetric and antisymmetric electron and hole states, while the other two, $|2\rangle=|S A\rangle$ and $|3\rangle=|A S\rangle$, remain dark. As $d$ increases, the QDM asymmetry and the Coulomb interaction mix these symmetric combinations as is clearly seen from the oscillator strengths (proportional to radiative rates), Fig. 3(b). Finally, in the limit of large $d$ the two QDs become isolated (no tunneling) and bright states are $|1\rangle=$ $|L L\rangle$ and $|2\rangle=|R R\rangle$ formed from electrons and holes both localized on the left and on the right dot, respectively. The energy splitting between them is $\Delta_{e}+\Delta_{h}$, where $\Delta_{e}\left(\Delta_{h}\right)$ is the electron (hole) asymmetric splitting due to the slight difference between the QDs. The other two, $|3\rangle$ and $|4\rangle$, are spatially indirect exciton states which are dark and split off by the Coulomb energy $E_{C}$. In contrast, in a symmetric QDM the two bright states would be separated by the Coulomb energy [9].
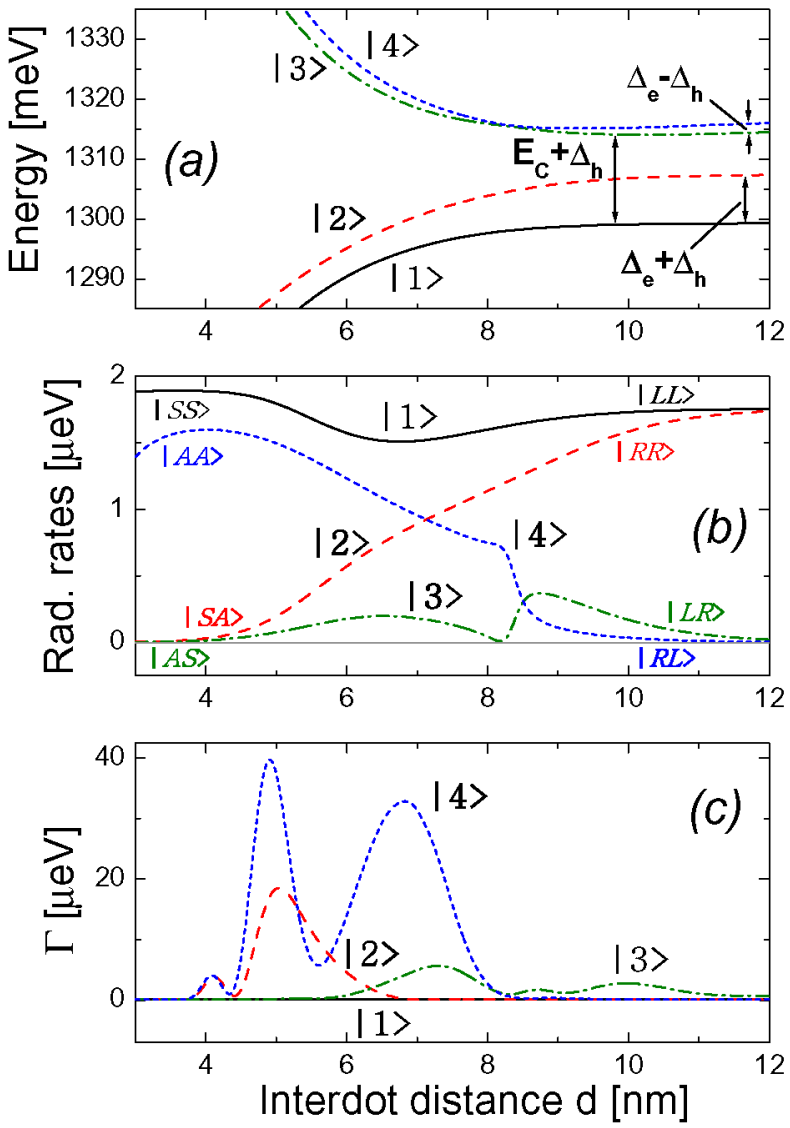

FIG. 3 (color online). Exciton energies (a) and radiative rates $\gamma_{n}^{\mathrm{rad}}=n_{r}(4 / 3)(\omega / c)^{3}\left|\mu_{n}\right|^{2}$ (b) calculated within the four-level model of InGaAs QDM accounting for the Coulomb interaction and $2 \%$ of asymmetry. (c) Exciton dephasing rates (ZPL widths $\Gamma_{n}$ ) of the QDM calculated at $T=10 \mathrm{~K}$. The meaning of $E_{C}$, $\Delta_{e}$, and $\Delta_{h}$ are given in the text.

The full linear polarization of a QDM is calculated up to second order in the cumulant expansion [16], using the described four-level excitonic model. Its Fourier transform, i.e., the absorption, shown in Fig. 4, contains four finite-width Lorentzian lines on the top of broadbands. The width of the broadband is of the order of the typical energy of phonons participating in the transitions, $\omega_{0} \sim 2 \mathrm{meV}$. Thus, if two levels come close to each other and the broadbands start to overlap, the ZPLs get considerably wider, due to real phonon-assisted transitions between neighboring levels. In QDMs this important mechanism of the dephasing is controlled by the tunneling which induces a level repulsion at short interdot distances.

However, there is another effect which leads to a quite unexpected result: Coulomb anticrossing. As $d$ increases, the two higher levels come close to each other and should exchange phonons more efficiently. Nevertheless, when the anticrossing is reached at around $d=8 \mathrm{~nm}$ (Fig. 4), the ZPL width suddenly drops and never restores at larger $d$. This is due to a change of the symmetry of states, owing to the Coulomb interaction. At $d>8 \mathrm{~nm}$ states $|3\rangle$ and $|4\rangle$ 


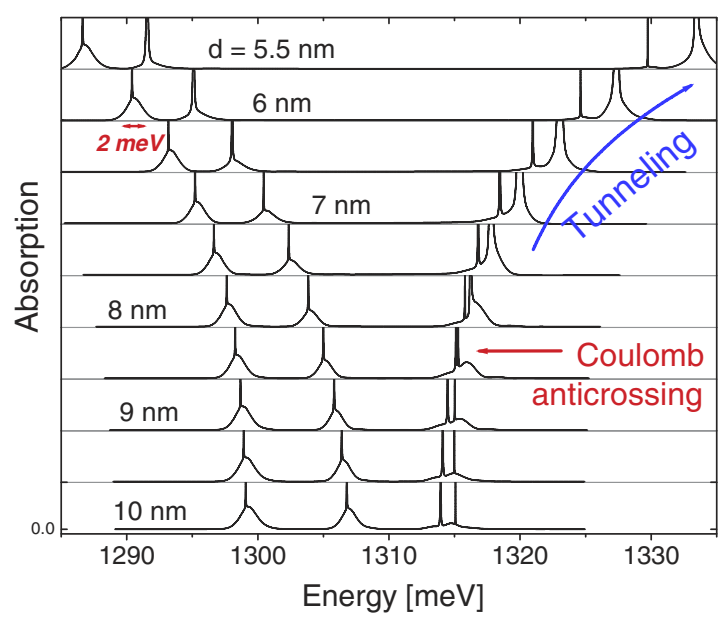

FIG. 4 (color online). Absorption spectrum (linear scale) of an asymmetric InGaAs QDM calculated at $T=10 \mathrm{~K}$ for different interdot distances $d$. The peaks of the ZPLs are truncated.

become more like $|L R\rangle$ and $|R L\rangle$, respectively [See Fig. 3(b)], and the exciton-phonon matrix element between them drops quickly by symmetry. Thus, real transitions between states $|3\rangle$ and $|4\rangle$ are not allowed any more and their dephasing is only due to virtual transitions into states $|1\rangle$ and $|2\rangle$.

Dephasing results for all four states are summarized in Fig. 3(c). Apart from the features already discussed, there are also oscillations in $\Gamma$ clearly seen for levels $|2\rangle$ and $|4\rangle$ in the region between $d=4 \mathrm{~nm}$ and $8 \mathrm{~nm}$. For an explanation, note that the matrix elements $M_{\mathbf{q}}^{n m}$, Eq. (2), are Fourier transforms of the electron (hole) probabilities. When located in different QDs, they carry a factor of $\exp (i q d)$. Since the typical phonon momentum participating in real transitions is $q_{0} \sim 1 / L_{z}$, one could expect that $\Gamma(d)$ has maxima spaced by a length of order $L_{z}$.

The temperature dependence of $\Gamma_{n}$ is shown in Fig. 5. At $d=6 \mathrm{~nm}$, levels $|3\rangle$ and $|4\rangle$ are already close to each other, and real phonon-assisted transitions between them are possible. As a result, the dephasing rates grow quickly with temperature. At the same time, levels $|1\rangle$ and $|2\rangle$ are far from each other and real transitions are suppressed. Still, virtual transitions contribute everywhere, with no strong dependence on level energies.

In conclusion, in the present microscopic approach to the dephasing in quantum dot molecules, we go beyond Fermi's golden rule and quadratic coupling model [8], by taking into account both real and virtual phononassisted transitions between exciton levels on an equal footing. We show that the broadening of the zero-phonon lines calculated for a few lowest exciton states depends strongly on interdot distance (via tunneling), electron-hole

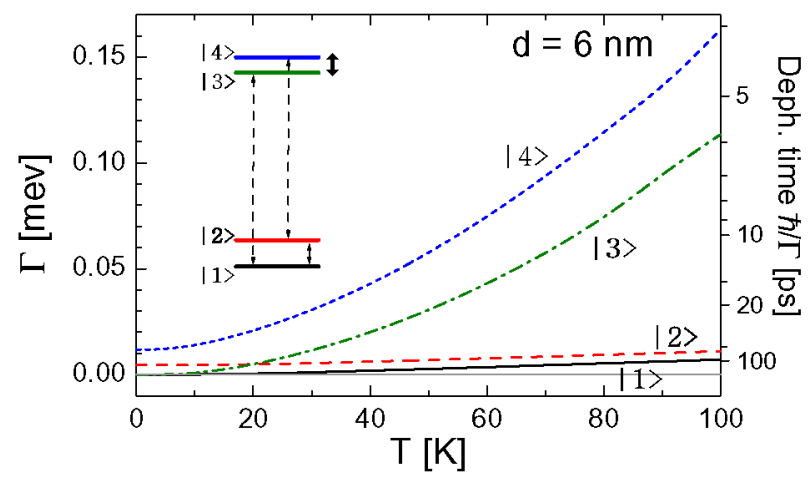

FIG. 5 (color online). Temperature dependence of the ZPL widths $\Gamma_{n}$ for the lowest four exciton levels in $d=6 \mathrm{~nm}$ QDM. Inset: real (virtual) phonon-assisted transitions between exciton states shown schematically by full (dashed) arrows.

Coulomb interaction, and asymmetry of the double-dot potentials.

Financial support by DFG Sonderforschungsbereich 296, Japan Society for the Promotion of Science (L03520), and the Russian Foundation for Basic Research (03-02-16772) is gratefully acknowledged.

*Electronic address: muljarov@gpi.ru

[1] M. Bayer et al., Science 291, 451 (2001).

[2] T. Calarco et al., Phys. Rev. A 68, 012310 (2003).

[3] G. Ortner et al., Phys. Rev. Lett. 94, 157401 (2005).

[4] P. Borri et al., Phys. Rev. Lett. 87, 157401 (2001).

[5] L. Besombes, K. Kheng, L. Marsal, and H. Mariette, Phys. Rev. B 63, 155307 (2001).

[6] G. Mahan, Many-Particle Physics (Plenum, New York, 1990).

[7] B. Krummheuer, V. M. Axt, and T. Kuhn, Phys. Rev. B 65, 195313 (2002); E. Pazy, Semicond. Sci. Technol. 17, 1172 (2002).

[8] E. A. Muljarov and R. Zimmermann, Phys. Rev. Lett. 93, 237401 (2004).

[9] B. Szafran, S. Bednarek, and J. Adamowski, Phys. Rev. B 64, 125301 (2001).

[10] P. Borri et al., Phys. Rev. Lett. 91, 267401 (2003).

[11] T. Takagahara, Phys. Rev. B 60, 2638 (1999).

[12] R. Kubo, J. Phys. Soc. Jpn. 17, 1100 (1962).

[13] S. Fafard et al., Appl. Phys. Lett. 76, 2268 (2000).

[14] Both lengths are connected to each other through the electron (hole) potential depth $U_{e(h)}: l_{e}^{4} m_{e} U_{e}=l_{h}^{4} m_{h} U_{h}$; $U_{e} / U_{h}=7 / 3$.

[15] Semiconductors, edited by O. Madelung, LandoltBörnstein New Series Vols. 17b and 22a (Springer, Berlin, 1986).

[16] The cumulant expansion converges well as long as the effective coupling $\left(M_{\mathbf{q}}^{n m}\right)^{2} N_{\text {Bose }}$ remains small. 